\title{
Evidence at the Centre Stage Bridging Theory-Practice Gap in Testing
}

\author{
Sadhana Parashar, Priyanka Sharma \\ ${ }^{1,2}$ National Testing Agency, Department of Higher Education \\ Ministry of Human Resource Development, India
}

\begin{abstract}
This paper discusses the significance and usage of variety of evidence from various sources, at different stages of educational testing to improve the quality of the test, especially validity of the decision drawn on the basis of the test scores about test takers. It is not limited to, but briefly touches the principle and practices of Evidence-Centered Design (ECD), and tries to establish linkages at various points of the testing cycle. While emphasizing the need of evidence-based approach of test development and interpretation of test scores, the paper also argues in favour of rigour-relevance balance. It extends to using evidence from measurement models from Classical Test Theory and Item Response Theory. However the intent is not just an academic review or psychometric analyses, but to produce usable evidence and present evidence in simpler form to users, who may be great subject experts and instruction designers or policy makers, but may not be great measurement experts.
\end{abstract}

Keywords: Evidence-based approach, Testing, Measurement Models 